\title{
EUS-guided portal pressure gradient measurement with a simple novel device: a human pilot study
}

\author{
Jason B. Samarasena, MD, Jason Y. Huang, FRACP, Takeshi Tsujino, MD, PhD, Daniel Thieu, BSc, \\ Allen Yu, BSc, Ke-Qin Hu, MD, John Lee, MD, Kenneth J. Chang, MD
}

\begin{abstract}
Background and Aims: Portal hypertension is a serious adverse event of liver cirrhosis. Recently, we developed a simple novel technique for EUS-guided portal pressure gradient (PPG) measurement (PPGM). Our animal studies showed excellent correlation between EUS-PPGM and interventional radiology-acquired PPGM. In this video we demonstrate the results of the first human pilot study of EUS-PPGM in patients with liver disease.
\end{abstract}

Methods: EUS-PPGM was performed by experienced endosonographers using a linear echoendoscope, a 25-gauge FNA needle, and a novel compact manometer. The portal vein and hepatic vein (or inferior vena cava) were targeted by use of a transgastric or transduodenal approach. Feasibility was defined as successful PPGM in each patient. Safety was based on adverse events captured in a postprocedural interview.

Results: Twenty-eight patients underwent EUS-PPGM with 100\% technical success and no adverse events. PPG ranged from 1.5 to $19 \mathrm{~mm} \mathrm{Hg}$ and had excellent correlation with clinical parameters of portal hypertension, including the presence of varices $(P=.0002)$, PH gastropathy $(P=.007)$, and thrombocytopenia $(P=.036)$.

Conclusion: This novel technique of EUS-PPGM using a 25-gauge needle and compact manometer is feasible and appears safe. Given the availability of EUS and the simplicity of the manometry setup, EUS-guided PPG may represent a promising breakthrough for procuring indispensable information in the management of patients with liver disease. (Gastrointest Endosc 2018;3:361-3.)

Portal hypertension $(\mathrm{PH})$ is a severe adverse event of liver cirrhosis. Clinical manifestations may include the formation of varices with associated GI bleeding, ascites, encephalopathy, or hepatorenal syndrome. ${ }^{1,2}$ Therefore, the diagnosis and quantification of PH by measuring portal pressure hold tremendous therapeutic and prognostic implications. ${ }^{2-4}$

The portal pressure gradient (PPG) is the difference between the portal vein (PV) pressure and the pressure within the hepatic vein (HV) (or inferior vena cava). It reflects the hepatic perfusion pressure. In patients with cirrhosis, portal pressure increases because of increased intrahepatic vascular resistance and increased portal blood flow. ${ }^{2}$

PPG is derived by subtracting the HV pressure from the PV pressure. These pressures ideally should be obtained through direct venous puncture. Currently, the PV pressure is not routinely measured. Instead, it is indirectly estimated by use of the wedged HV pressure (WHVP), which approxi- mates PV pressure in the cirrhotic liver. Thus, only the HV pressure is a true direct measurement. This gradient is termed the HV pressure gradient (HVPG), and it accurately reflects the degree of $\mathrm{PH}$ in all forms of sinusoidal and postsinusoidal causes of $\mathrm{PH}^{1,5,6}$

We have already described EUS-guided PPG measurement using a 25-gauge needle and a novel compact manometer in an animal model. ${ }^{7}$ Our method demonstrated excellent accuracy and a strong correlation with pressure values obtained by the criterion standard of transjugular wedged and free $\mathrm{HV}$ pressure measurements by interventional radiology. Here, we present the first pilot study in humans demonstrating safe and accurate direct PPG measurements without the need for ionizing radiation, transhepatic catheter placement, or surgery (Video 1, available online at www.VideoGIE.org).

EUS-PPG was performed at a single tertiary academic center by experienced endosonographers. All cases were 
performed with the patients under monitored anesthesia or general anesthesia and placed in the supine position. Patients between the ages of 18 and 75 with a history of liver disease or suspected cirrhosis were considered for PPG measurement.

The apparatus for PPG measurement included a linear echoendoscope (GF-UC140P-AL5; Olympus, Tokyo, Japan), a 25-gauge FNA needle (Cook Medical, Winston-Salem, NC, USA), and a compact manometer (Fig. 1) with noncompressible tubing (Cook Medical, Bloomington, Ind, USA). The compact manometer has a digital readout and measures $2 \mathrm{~cm} \times 3 \mathrm{~cm} \times 2 \mathrm{~cm}$, and it is currently not available commercially.

Before PPG measurement, an endoscopic evaluation was performed to evaluate for markers of $\mathrm{PH}$ such as varices and portal hypertensive gastropathy. The endoscopists were not blinded to clinical information before the PPG measurements. For PPG measurement, the patients were placed in a supine position, and the manometer was zeroed at the midaxillary line before echoendoscope insertion. Measurements were conducted in the PV (Fig. 2) and $\mathrm{HV}$ (Fig. 3) where possible. If the $\mathrm{HV}$ was inaccessible because of anatomic limitations, the inferior vena cava was targeted. PV manometry was performed through a transgastric or a transduodenal (1 patient), transhepatic approach, and only the intrahepatic portion near the PV bifurcation was accessed. A small amount (1 $\mathrm{mL})$ of heparinized saline solution $(100 \mathrm{IU} / \mathrm{mL})$ was flushed through the primed FNA needle (no stylet) before each EUS reading. After 30 to 60 seconds of pressure stabilization, the reading was recorded. Three separate readings per vessel were performed, and a mean pressure was calculated. On withdrawal of the needle, just before leaving the liver capsule, color Doppler was used to ensure absence of flow in the needle track. Intraprocedural prophylactic antibiotics were given.

A total of 28 patients underwent portal pressure manometry in this retrospective study, and pressures were successfully achieved in all 28 patients. Eighteen patients were male; conditions and indications included viral hepatitis (15 patients), alcohol use (6), increased liver function test results (5), and nonalcoholic fatty liver disease (2). There were 16 patients with a platelet count less than 150, and 4 patients had International Normalized Ratios $>1.2$.

PPG values ranged from 1.5 to $19 \mathrm{~mm} \mathrm{Hg}$ (mean, $8.2 \mathrm{~mm} \mathrm{Hg}$ ). Fifteen of 28 patients (57.1\%) had evidence of PH based on PPG, of whom 10 of 15 (66.7\%) had clinically significant portal hypertension (CSPH). Eleven of 28 study participants had endoscopic evidence of either esophageal or gastric varices, with all 11 (100\%) having $\mathrm{PH}$ and 10 (90.9\%) patients having CSPH based on EUSPPG measurement.

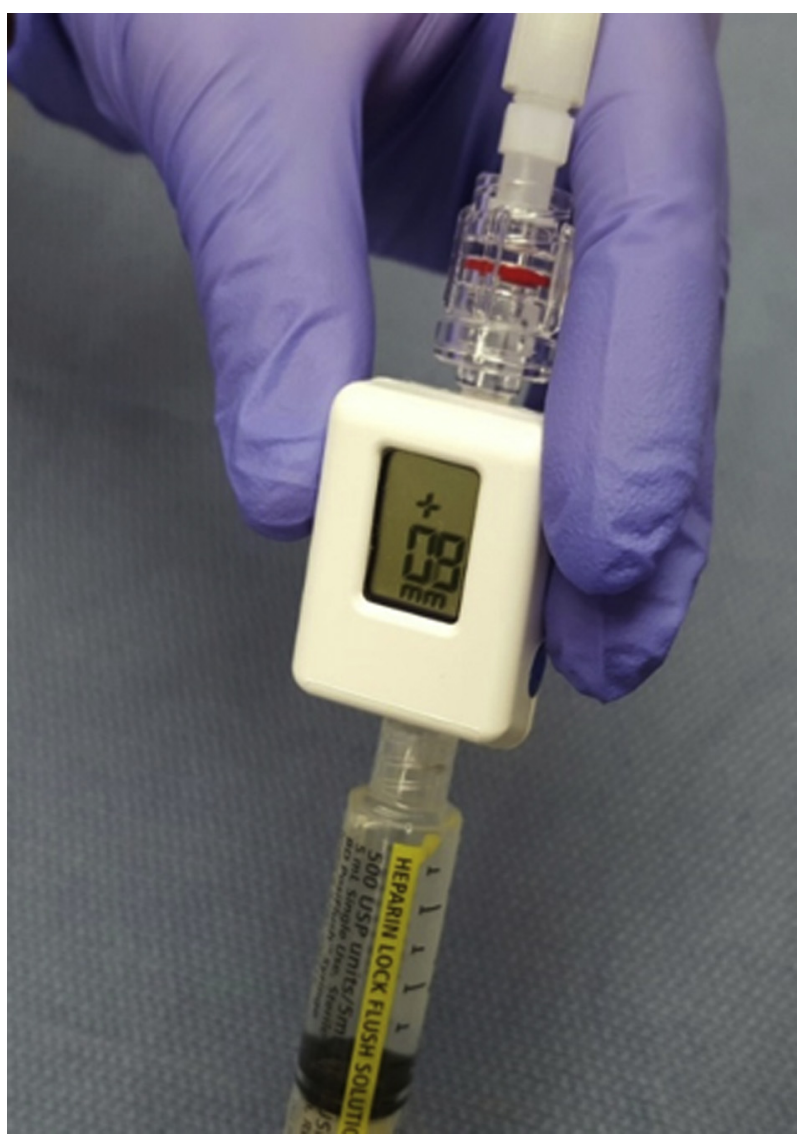

Figure 1. Compact manometer.

No intraprocedural or postprocedural adverse events such as bleeding, perforation, or pain were seen in any patient. There were no infectious adverse events. There was excellent association between PPG and clinical parameters. $^{8}$

Limitations to the study include its retrospective nature. There was also no criterion standard for correlation such as interventional radiology-acquired pressure measurements. This study demonstrates that EUS-guided portal pressure measurement by use of a 25 -gauge needle and a novel compact manometer is feasible and appears safe in humans. There were no technical failures with PPG manometry, and there were no adverse events in any patient.

\section{DISCLOSURE}

Dr Samarasensa and Dr Lee are the recipients of educational grants from Cook Medical. Dr Chang is a consultant with Cook Medical. All other authors disclosed no financial relationships relevant to this publication. 

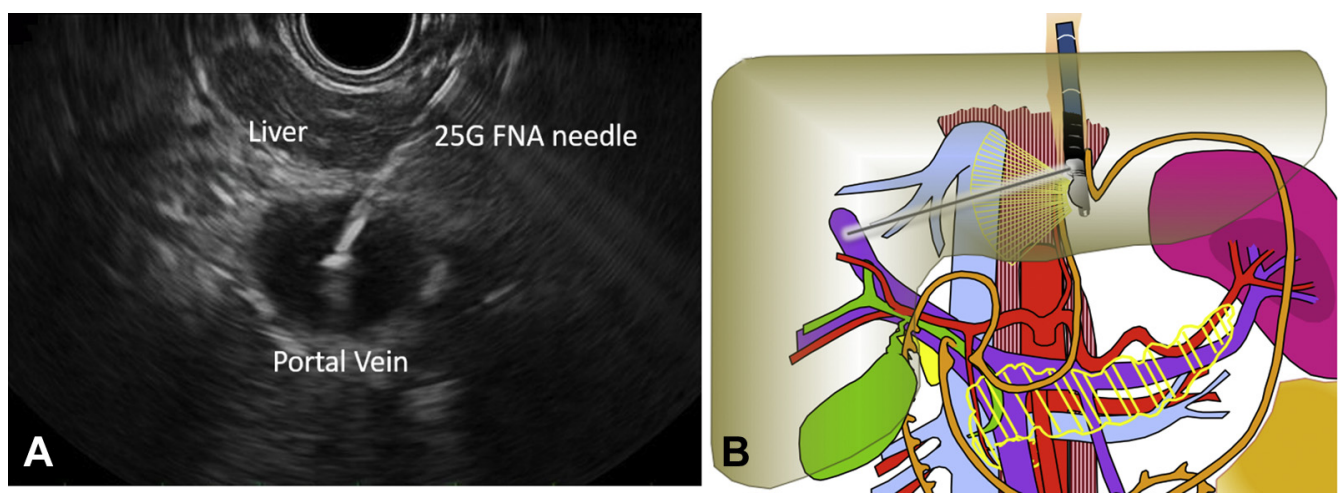

Figure 2. A, EUS image of transgastric transhepatic needle puncture into the portal vein with a 25-gauge FNA needle. B, Representation of EUS-guided transgastric portal vein puncture.
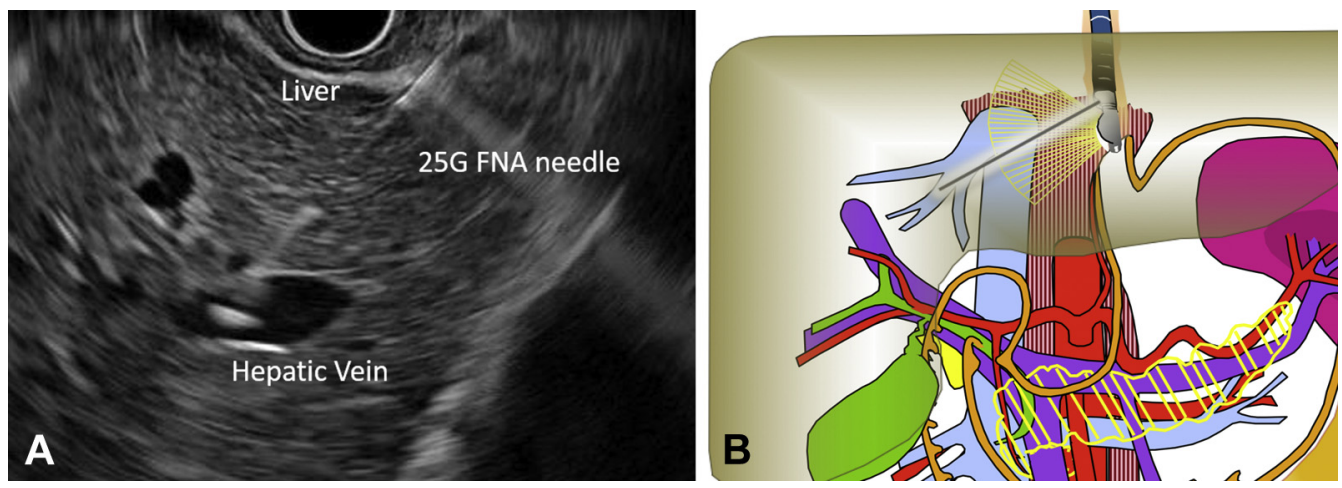

Figure 3. A, EUS image of transgastric transhepatic needle puncture into the hepatic vein with a 25-gauge FNA needle. B, Representation of EUS-guided transgastric hepatic vein puncture.

Abbreviations: $H V$, hepatic vein; HVPG, HV pressure gradient; $P H$, portal hypertension; PPG, portal pressure gradient; PPGM, PPG measurement; $P V$, portal vein; WHVP, wedged HV pressure.

\section{REFERENCES}

1. Thalheimer $U$, Bellis $L$, Puoti $C$, et al. Should we routinely measure portal pressure in patients with cirrhosis, using hepatic venous pressure gradient (HVPG) as a guide for prophylaxis and therapy of bleeding and rebleeding? No. Eur J Intern Med 2011;22:5-7.

2. Sanyal AJ, Bosch J, Blei A, et al. Portal hypertension and its complications. Gastroenterology 2008;134:1715-28.

3. Burroughs AK, McCormick PA. Natural history and prognosis of variceal bleeding. Baillieres Clin Gastroenterol 1992;6:437-50.

4. D'Amico G, Garcia-Tsao G, Pagliaro L. Natural history and prognostic indicators of survival in cirrhosis: a systematic review of 118 studies. J Hepatol 2006;44:217-31.

5. Armonis A, Patch D, Burroughs A. Hepatic venous pressure measurement: an old test as a new prognostic marker in cirrhosis? Hepatology 1997;25:245-8.
6. Groszmann RJ. Reassessing portal venous pressure measurements, Gastroenterology 1984;86:1611-4.

7. Huang JY, Samarasena JB, Tsujino T, et al. EUS-guided portal pressure gradient measurement with a novel 25-gauge needle device versus the standard transjugular approach: a comparison animal study. Gastrointest Endosc 2016;84:358-62.

8. Huang JY, Samarasena JB, Tsujino T, et al. EUS-guided portal pressure gradient measurement with a simple novel device: a human pilot study. Gastrointest Endosc 2017:85:996-1001.

H. H. Chao Comprehensive Digestive Disease Center, Division of Gastroenterology and Hepatology, University of California, Irvine, Orange, California, USA.

Copyright (c) 2018 American Society for Gastrointestinal Endoscopy. Published by Elsevier Inc. This is an open access article under the CC BYNC-ND license (http://creativecommons.org/licenses/by-nc-nd/4.0/).

https://doi.org/10.1016/j.vgie.2018.07.013 